An official journal of the / Un journal officiel de la "Société Sénégalaise de Cancérologie" (SOSECAN)

Journal homepage: www.africanjournalofoncology.com

\title{
Place of non-drug treatments in the management of pain in cancerology
}

\section{Place des traitements non médicamenteux dans la gestion de la douleur en cancérologie}

\author{
S. Ka ${ }^{\prime *}$, J. Thiam', S. Baldé', PS. Dieng', ME. Charfi', MM. Dieng', PM. Gaye', A. Dem'.
}

I Institut Joliot Curie de Dakar, Sénégal.

Le cancer se caractérise par une invasion tissulaire entraînant destruction, inflammation, irritation, obstructions, nécroses, hémorragies et compressions. Toutes ces causes de douleur peuvent être présentes à des degrés divers à tous les stades. La prise en charge de la douleur dépend donc de la prise en charge du cancer et vice versa.

La destruction tissulaire est la règle dans les cancers des organes solides, dans des circonstances primitives ou métastatiques. La douleur est généralement multifactorielle.

La gestion de cette douleur se fait à différents niveaux. Elle est essentiellement médicamenteuse intégrant plusieurs paliers [I]. La morphine, palier supérieur, quasi indispensable, est caractérisée par des effets secondaires notoires comme la constipation, la dépression respiratoire et l'accoutumance [2]. Les traitements non médicamenteux de la douleur intègrent des traitements spécifiques des cancers et des thérapeutiques novatrices et prometteuses. La physiothérapie, longtemps controversée comme utile dans les cancers, est de plus en plus reconnue comme essentielle après une bonne évaluation du cancer et des traitements [3, 4]. L'acupuncture n'a pas montré une réelle efficacité dans la gestion de la douleur [5]. Des approches cognitivo-comportementales comme la relaxation ont montré une efficacité significative dans le traitement de la douleur chronique. Elles sont cependant peu évaluées dans les douleurs néoplasiques [6].

Les traitements spécifiques du cancer participent au contrôle de la douleur indirectement lors du contrôle de la maladie ou directement dans le contrôle des lésions principalement secondaires. C'est le cas de la radiothérapie des métastases cérébrales. Le diagnostic de métastase est fait par scanner ou imagerie par résonance magnétique (IRM) devant le syndrome d'hypertension crânienne. Les effets des rayonnements sont tumoricides, anti-inflammatoires, décongestionnants, mais aussi pour la consolidation et la décompression neurologique. Son efficacité analgésique est très importante. L'utilisation d'une perfusion intrathécale délivrée par pompe chez des patients sélectionnés devrait être une option complémentaire [7].

Les tumeurs osseuses, plus souvent secondaires que primitives, révélatrices de cancers du fait de la douleur offrent un choix de traitement plus large. Même si à ce stade la guérison est impossible. Le traitement repose sur des outils non spécifiques comme les antalgiques, les bisphosphonates, la cimentoplastie et la kinésithérapie mais aussi des outils spécifiques comme la radiothérapie. L'acide zolédronique et le dénosumab restent un choix sûr et efficace chez les patientes ménopausées non métastatiques agressives atteintes d'un cancer du sein et chez les patientes métastatiques osseuses [8]. La radiothérapie joue un rôle fondamental avec une efficacité antalgique remarquable dans la douleur associée aux métastases osseuses en général et en particulier dans les métastases vertébrales chez 30 à $80 \%$ des patients [9]. Dans ce domaine, de nouvelles découvertes thérapeutiques comme le strontium-89 sont très prometteuses dans le contrôle de la douleur en particulier dans les traitements spécifiques résistants tels que la castration dans le cancer de la prostate [10].

Le cancer inflammatoire est une affection courante. Le cancer a la capacité d'induire une inflammation par voie génétique ou infectieuse et par progression tumorale. Le cancer du sein inflammatoire et les cancers obstructifs ou ulcérés en sont de bonnes illustrations. La chimiothérapie, I'hormonothérapie ou les thérapies ciblées sont à la pointe du traitement de la douleur. La chirurgie par levée d'obstacles et élimination des lésions joue un rôle indirect [I I].

La douleur peut être spécifique à l'organe de par sa structure, son fonctionnement, sa position anatomique et son innervation [12]. La douleur évoque le diagnostic. Le cancer du pancréas en est un exemple particulier. Le type de douleur solaire est typique. Le traitement de cette douleur est difficile. Les opioïdes sont inefficaces et la chirurgie est limitée par la découverte tardive de la tumeur, la position anatomique et la profondeur de l'organe. La chimiothérapie et la radiothérapie ne sont pas efficaces [13]. Le bloc chirurgical, l'alcoolisation et les neurotomies splanchniques de même que la neurostimulation dans d'autres cas restent souvent les derniers recours [14].

Les traitements anticancéreux spécifiques tels que la chirurgie, la radiothérapie et les traitements médicaux de même que les traitements novateurs non médicamenteux comme la physiothérapie et les traitements cognitivo-comportementaux offrent des avantages considérables dans le traitement de la douleur cancéreuse. Leur combinaison semble être la voie de salut dans le traitement des douleurs chez le patient atteint de cancer.

\section{CONFLITS D'INTERET}

Les auteurs n'ont déclaré aucun conflit d'intérêts.

\section{REFERENCES}

*Corresponding author: Email : sidy.ka@ucad.edu.sn. 
I. Portenoy RK. Treatment of cancer pain. Lancet 20I I; 377: 2236-2247.

2. Steins MB, Eschbach C, Villalobos M, Thomas M. Pain management in palliative care. Pneumologie 20I7;7I (5):297-306.

3. Gomez I, szecanecz É, Szekanecz Z. Physiotherapy of cancer patients. Orv Hetil. 2016;157(3I):|224-3|.

4. Lee Sh, Kim JY, Yeo S, Kim SH, Lim S. Meta-analysis of massage therapy on Cancer Pain. Integr Cancrt Ther. 2015; 14(4):297-304.

5. Paley CA, Johnson MI, Tashani OA, Bagnall AM. Acupuncture for Cancer pain in adults. Cochrane database Syst Rev. 2015;2015(I0):CD007753.

6. Knoerl R, Lavoie Smith EM, Weisberg J. Chronic pain and behavioral therapy: An integrative review. West J Nurs Res. 2016;38(5):596-628.

7. Dupoiron DA, Lefebvre-kuntz DB, Brenet OA, De Bourmont SA, Grelon FA, et al. Refractory cancer pain and intrathecal infusion: Experience of three cancer management centers. Douleurs 20 I I; 12: 140146.

8. Chen F, Pu F. Safety of denosumab versus zoledronic acid in patients with bone metastases: A meta-analysis of randomized controlled trials. Oncol Res Treat 2016; 39: 453-459.

9. Lagrange JL, Pan C, Calitchi E, Diana C, Muresan M, et al. Practical radiation therapy for painful bone metastases. Rev Rhumat 2008; 75: 530-535.

10. Laing $A H$, Ackery DM, Bayly RJ, Buchanan RB, Lewington $\mathrm{VJ}$, et al. Strontium-89 chloride for pain palliation in prostatic skeletal malignancy. $\mathrm{Br}$ J Radiol 1991; 64: 816-822.

II. Bertucci F, Finetti P, Rougemont J, Charafe-Jauffret E, Nasser V, et al. Gene expression profiling for molecular characterization of inflammatory breast cancer and prediction of response to chemotherapy. Cancer res 2004; 64: 8558-8565.

12. Kahan B. Cancer pain and current theory for pain control. Phys Med Rehabil Clin N Am 2014; 25: 439456.

13. Mondo EL, Noel MS, Katz AW, Schoeniger LO, Hezel AF. Unresectable locally advanced pancreatic cancer: Treatment with neoadjuvant leucovorin, fluorouracil, irinotecan, and oxaliplatin and assessment of surgical resectability. J Clin Oncol 2013; 3: e37-e39.

14. Fontaine $\mathrm{D}$, Blond $\mathrm{S}$, Mertens $\mathrm{P}$, Lanteri-Minet $\mathrm{M}$. Neurosurgical treatment of chronic pain. Neurochirurgie 2015; 61: 22-29. 